\title{
Psychodrama goes digital!
}

\section{Erste Schritte in der Gruppenselbsterfahrung mit Hilfe von Instant-Messaging, E-mail und Videokonferenzen in Zeiten der Covid-19-Pandemie}

\section{Ulrike Altendorfer-Kling}

Online publiziert: 11. August 2020

(C) Springer Fachmedien Wiesbaden GmbH, ein Teil von Springer Nature 2020

Zusammenfassung Dieser Beitrag der Zeitschrift für Psychodrama und Soziometrie (ZPS) setzt sich mit der Problematik auseinander, dass für die Gruppenselbsterfahrung als einem unerlässlichen Element im Rahmen der Ausbildung zum oder zur Psychodrama-PsychotherapeutIn, die Fertigkeiten für intensive Nutzung digitaler Medien nun im Zuge der Covid-19-Pandemie sehr unerwartet gebraucht werden. Wenn wir uns nicht mehr in der physischen Gruppe treffen dürfen, dann stellen diese Medien genau die entscheidende Option dar, wie Psychotherapie- und Ausbildungsgruppen weitergeführt werden können. In Krisenzeiten ist es für uns Menschen besonders wichtig, den Kontakt zueinander aufrechterhalten, einander zu unterstützen und voneinander lernen zu können. In diesem Beitrag wird eine PsychodramaSelbsterfahrungsgruppe in Psychotherapieausbildung vorgestellt, die sich bis zur Einführung der restriktiven Maßnahmen durch die Bundesregierung Österreichs mit Versammlungsverboten von mehr als fünf Personen, für vierzig Ausbildungseinheiten getroffen hat. Die Folgetermine wurden nun via digitale Medien durchgeführt. Ich gebe einen Vorschlag, wie Psychodrama in Zeiten von Covid-19 erlernt und wirksam werden kann.

Schlüsselwörter Digitale Medien · Instant Messaging · Videotelefonie · Psychodrama · Gruppenselbsterfahrung · Covid-19 · Traumatherapie · Krisenintervention · Erwachsene

Dr. med. univ. U. Altendorfer-Kling ( $\square)$

Dr. U. Altendorfer-Kling, Praxis für Psychodramapsychotherapie, Mönchsberg 33, 5020 Salzburg,

Österreich

E-Mail: praxis@draltendorfer.at 


\section{Psychodrama goes digital!}

Get started in the group self-experience with the help of instant messaging, e-mail and videoconferencing in times of the Covid-19 pandemic

Abstract This contribution to the Zeitschrift für Psychodrama und Soziometrie (ZPS) deals with the fact that for Group self-experience, which is an essential part of the psychotherapy training, suddenly the intensive use of digital media has overwhelmed most of us in times of the Covid-19 pandemia. If we are no longer allowed to meet physically, these media show us the decisive option they offer for continuation of the psychotherapy- and training-groups. In the crisis it is most important for us human beings to keep in contact, support each other and learn from each other. This contribution presents a Psychodrama-Selfexperience Group, whose participants met for 40 training units until the restrictions of the Austrian Government with ban on assembly of more than 5 persons have been introduced. The following training sessions continue via digital media. The article wants to show an idea of how Psychodrama can still be effective and learned in times of Covid-19.

Keywords Digital media - Instant messaging · Video telephony · Psychodrama · Group self-experience · Covid-19 · Traumatherapy · Crisis Intervention · Adults

\section{Ausgangslage und Gruppenprozess}

Um die Unterbrechung der bereits laufenden und gut arbeitenden Selbsterfahrungsgruppe im Fort- und Weiterbildungskontext zu vermeiden, wurde kurz nach Inkrafttreten des Versammlungsverbots durch die Österreichische Regierung der Modus vivendi auf digitale Medien umgestellt. Die Gruppe traf sich nun laufend im Rahmen einer den meisten LeserInnen aus dem Privatleben bekannten Instant Messaging-Gruppe. Die genutzte Instant-Messenger-Applikation bietet für Smartphones mit End-zu-End-Verschlüsselung gesicherte Kommunikation innerhalb der Gruppe. Diese ermöglicht über Internet das kostenlose Senden und Empfangen von Textnachrichten, Bildern, Videos und Audiobotschaften. Die zehn Gruppenmitglieder nützten die Schreibfunktionen und Möglichkeiten, Hyperlinks und Videos anzuhängen und gaben einander viel Rückhalt, Informationen und Sharings, indem sie ihren Alltag ausschnittweise miteinander teilten. So entstand parallel zum Trainingsinhalt mitlaufend ein Fluss von sehr persönlichen Rückmeldungen über einen längeren Zeitraum. Dieser Fluss wurde durch Eingabefristen nicht behindert.

$\mathrm{Zu}$ Beginn der digitalen Seminarführung gab die Gruppenleitung Inputs für die Erwärmung zur aktuellen Situation. Zunächst fand, wie in konventionellen Gruppentreffen auch, eine schriftliche Begrüßung der GruppenteilnehmerInnen durch die Gruppenleitung mit einer Standortbestimmung statt. Die im Kreis sitzenden GruppenteilnehmerInnen musste man sich vorstellen und die Begegnungsbühne wurde mit der Ankündigung für das weitere Warm Up, das mit Unterstützung von verschiedenen Fotomotiven durchgeführt werden würde, geschlossen. Die neun Bilder wurden in die Instant Messaging Gruppe gestellt und konnten von allen Gruppenmitgliedern gleichzeitig gesehen werden. Sie regten zur Selbstreflexion auf der inneren 
Bühne an und lösten einen Fluss von teilweise geschriebenen Darstellungen der aktuellen Erlebnisse und dazugehörigen Empfindungen aus, von den TeilnehmerInnen wurden auch selbstgestaltete Videos geschickt, einige Collagen oder auf andere Weise künstlerisch gestaltete bildhafte Darstellungen und stimmungsvolle Fotos. Diese regten zu schriftlichen Kommentaren und Diskussionen an. Die Funktion einer Audiobotschaft wurde nicht genützt. An psychodramatischen Interventionstechniken wurde vor allem stützendes und aktivierendes Doppeln und verbales Spiegeln auf diese Weise umgesetzt und im Rahmen der Selbstreflexionen zum inneren Monolog angeregt. Teilweise wurden außerdem Symbolisierungstechniken umgesetzt und die Skripttechnik mithilfe von Gedichten und Liedern verwendet, um die Erlebnisse zu erfassen und zu benennen. Dies diente zur Förderung von Spontaneität, Kreativität und Rollenflexibilität innerhalb der Gruppe.

Im konventionellen Psychodrama-Setting wäre nun der richtige Zeitpunkt, aus diesen Inputs, Szenen für ProtagonistInnenspiele oder Soziodramen zu erarbeiten. In der aktuellen Situation wurde das Gruppenthema „Angst versus Hoffnung und Zuversicht" aufgegriffen und eine weitere Reflexion zur aktuellen gesellschaftlichen Situation angeleitet. Die Gruppenmitglieder freuten sich schon sehr darauf, einander hoffentlich bald wiederzusehen und wünschten einander wiederholt, gesund zu bleiben. So wurde immer wieder der Fokus in die Zukunft und auf eine bessere Situation gelegt. Es wurde Einfühlung in und Empathie für den oder die Nächste angeregt und gleichzeitig auch die Einfühlung für sich selbst trainiert. Viele Gruppenmitglieder holten sich Zuversicht aus der Natur und genossen den Frühling mehr denn jäh mit allen Sinnen. Tage wurden strukturiert und lange Aufgeschobenes erledigt. Sehr persönliche Schicksalsschläge wurden ebenfalls thematisiert sowie entspannende und freudige Ereignisse. Eine Selbstbesinnung mitten in all dem Trubel fand statt, mit deren Hilfe die Gruppenmitglieder einen Weg suchten, sich auf die neue Situation einzustellen. Manche verharrten in dem empfundenen Chaos zunächst schweigend und wortkarg, weil es so schwerfiel, die passenden Worte zu finden.

Immer wieder äußerten die Gruppenmitglieder einander Dank und gegenseitige Wertschätzung für ihre Beiträge. So vermittelten sie einander stabilisierende glückliche Emotionen und Zuversicht in einer schwierigen Phase ihres Lebens. Jeder und jede trug auf seine und ihre persönliche Art dazu bei, sich an die plötzlichen Veränderungen in der aktuellen Lebenslage anzupassen und kreative Lösungen zu suchen. Es wurde immer wieder auf Ruhe, Geduld und Beziehungen hingewiesen und gegenseitige Hilfsangebote gemacht. Humorvolle und sehr berührende Beiträge wurden ebenfalls in die Gruppe gestellt.

\section{Warm Up}

Die durch das Warm-Up über die Selbstreflexionen ausgelösten Beiträge wurden nun zu einem Stimmungsbild unserer Gesellschaft weiterbearbeitet. Die Gruppenmitglieder verfassten asynchron Texte oder Collagen und Brainstorming-Zeichnungen oder Fotos in Einzelarbeit und stellten diese in die digitale Gruppe des Instant Messaging Services. Innerhalb der Gruppe wurden diese dann schriftlich über den Messaging 
Dienst kommentiert und die Themen der Einzelnen, aber auch das Gruppenthema ausgearbeitet.

Die zeitweise bewusste Karenz vom Medienkonsum und vom stetigen Informationsfluss ist ebenfalls ein Thema in der Gruppe gewesen. Manchen fiel es schwer, die laufend eingehenden Nachrichten erst zum individuell passenden Zeitpunkt zu lesen und eventuell zu reagieren. Sie fühlten sich dann durch das wiederkehrende Signalgeräusch gestört und gestresst. Andere gaben ihre Beiträge einfach zu bestimmten Zeitpunkten ab und kommentierten die bisherigen Nachrichten gesammelt.

\section{Gesellschaftsthemen}

Das Gruppenthema entwickelte sich hin zum Aufzeigen der dialektischen Prozesse unseres Lebens und den Ambivalenzen, die wir dabei auszuhalten haben. Unter uns ÄrztInnen und PsychotherapeutInnen kamen Fragen auf, wie wir unsere PatientInnen beruhigen und ihnen ihre Ängste nehmen können und zugleich uns selbst vor emotionaler und Reizüberflutung schützen können.

\section{Spiel/Aktionsphase}

Das Angebot der Videotelefonie im 2. Wochenendseminar wurde unterschiedlich gern angenommen. Es fiel zeitlich in die Reaktionsphase der Covid-19-Krise mit verschiedenen hochkochenden Emotionen der Gruppenmitglieder, teilweise traten Verweigerungstendenzen auf.

Während des ersten Videomeetings an diesem 2. Seminarwochenende wurde nach der Begrüßungsrunde mit deutlich sichtbarer Wiedersehensfreude der Beteiligten und dem Warm-Up von der Seminarleitung die psychodramatische Kriseninterventionstechnik des „Roten Fadens der Zeit“" nach Stadler et al. (2018) als Symbolarbeit auf der Tischbühne vorgestellt. Die Gruppenleitung bot einen kleinen Tisch mit Symbolen an und auf einem zweiten Tisch legte sie nach Rücksprache mit den Gruppenmitgliedern die verschiedenen Elemente wie den roten Faden der Zeit, das Symbol für den Beginn der Krise und die weiteren gemeinsam via Videokonferenz angesprochenen Themen auf. Die Gruppenmitglieder konnten sich mit ihren Fragen und Überlegungen zu dieser Technik einbringen. So wurde gemeinsam die Szene gestaltet.

In einem zweiten Schritt wurde die individuelle Rollenwahl im Soziodrama des Lebens mit dem Titel Selbsterfahrungsgruppe in der Covid-19-Krise (Ort und Zeit der Handlung: Österreich, Frühjahr 2020) bewusst gemacht.

Wir handelten nach dem Grundsatz von William Shakespeare (1599) „Die ganze Welt ist eine Bühne und alle Frauen und Männer bloße Spieler.“ - Daher holten wir das aktuelle Soziodrama der Covid-19-Krise aus der Welt in unsere Gruppe herein und reflektierten gemeinsam.

Die verschiedenen antagonistisch eingenommenen Rollen sind die der HelferIn - Betroffene(r), der oder die BewahrerIn - VisionärIn, KämpferIn - TotstellerIn, Flüchtende(r) - StrategIn, Ängstliche, Wütende, Essende, um nur einige zu nennen. 
Es wurden folgende Fragen beantwortet: Welche Rolle möchte ich in diesem Soziodrama des Lebens spielen? Was hindert mich sie auszuführen? Was wäre dabei hilfreich? Wen kann ich um Hilfe bitten, falls ich diese benötige, um meine Rolle klarer ausführen zu können? Gemeinsam wurde erarbeitet, welche Rollenwahl hilfreich erscheint und was es dazu braucht, die gewählte Rolle zu spielen und durchzuhalten. Es wurde ein Bewusstsein für die Bedeutung von Psychodrama in der Krisenbewältigung und Resilienzentwicklung geschaffen.

Jedes Gruppenmitglied hatte nun die Möglichkeit des inneren Rollenwechsels in die Antagonistenrolle, um eine Empathie für Mitmenschen in der entsprechenden Rolle zu entwickeln. Die bewusste Rollengestaltung wurde durch die Verschriftlichung nach und die Besprechung im Rahmen der Videokonferenz intensiviert.

Die Seminarleitung schickte der Gruppe nach der ersten Videositzung eine schriftliche Zusammenfassung des Lehrinhaltes mit den oben genannten Fragen, einer Anregung zur Selbstreflexion mithilfe des „Roten Fadens der Zeit“ nach Stadler et al. (2018) und soziometrischen Skalierungsfragen zur Erfassung der Gruppendynamik per E-Mail, um die individuellen Erfahrungen klarer auszuformulieren und zu vertiefen. Unter anderem wurde eine soziometrische Einstufung der einzelnen Gruppenmitglieder zu einem Zeitpunkt vor und während der Covid-19-Krise zu den Kriterien „Emotionale Beteiligung am Gruppengeschehen“ und „Gruppenzugehörigkeit“ mittels eines Koordinatensystems mit der Skalierung von -10 bis +10 erfragt (Abb. 1).

Abb. 1 Skalenarbeit mit Koordinatensystem

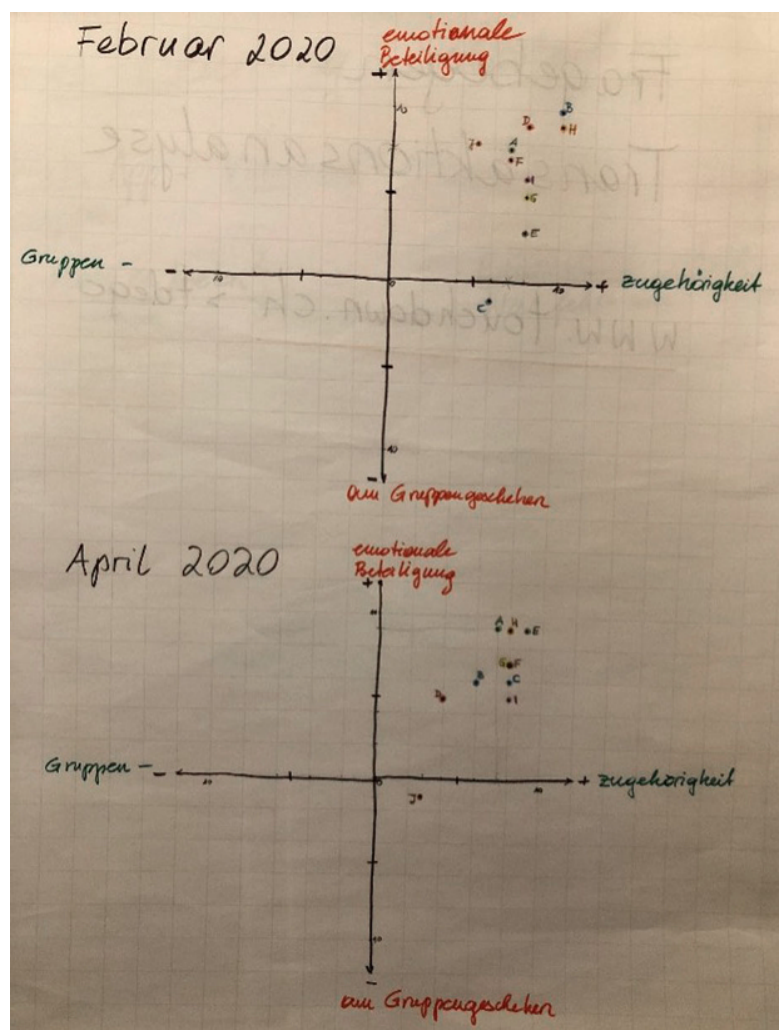


Die emotionale Beteiligung wurde zum ersten Zeitpunkt mit hohen positiven Werten um die +10 skaliert, zum zweiten Zeitpunkt mit etwas schwächer positiven, aber von den meisten Gruppenmitgliedern immer noch über +5 bewertet. Ihre Gruppenzugehörigkeit skalierten die TeilnehmerInnen zum ersten Zeitpunkt mit Werten höher als +6 (die meisten, $60 \%$, lagen bei +8 und +10 ) und zum 2. Messzeitpunkt mit einem maximalen Wert bei +9 , wobei die meisten, $40 \%$, sich bei +8 einstuften. Je eine Person lag zu beiden Zeitpunkten mit ihrer emotionalen Beteiligung bei -1 bei positiver Einstufung der Gruppenzugehörigkeit.

Dieses Skalierungsergebnis hat mich positiv überrascht, weil ich im Rahmen der Diskussionen erwartet hätte, dass sich die Veränderungen aufgrund der Covid19-Krise stärker auf die Emotionalität und die Gruppenzugehörigkeit auswirkten. Die einzelnen Personen machten teilweise individuell starke Schwankungen durch, jedoch, die Gruppe als solche blieb zum zweiten Zeitpunkt erfreulicherweise stabil im positiven Skalenbereich mit Werten über +5 .

\section{Sharing/Rollenfeedback/Integration}

Die zweite Videositzung fand fünf Tage später zur Beendigung des Seminars statt, damit genügend Zeit für die selbstreflexiven Prozesse der Gruppenmitglieder eingeplant werden konnte. Sie diente dem Mitteilen der Sharings der GruppenteilnehmerInnen, Rollenfeedback und Diskussion der aktuellen Erlebnisse und Erkenntnisse und zur Integration der Erfahrungen. Zur besseren zeitlichen Orientierung wurde der Gruppenprozess mittels Abb. 2 veranschaulicht und die rote Markierung immer wieder an der entsprechenden Stelle angebracht.

\section{Möglichkeiten und Hindernisse}

In der Gruppe wurde der Vorteil der asynchronen Kommunikation darin gesehen, dass jeder und jede dann schreiben und Bilder, Videos oder Audios in die Gruppe

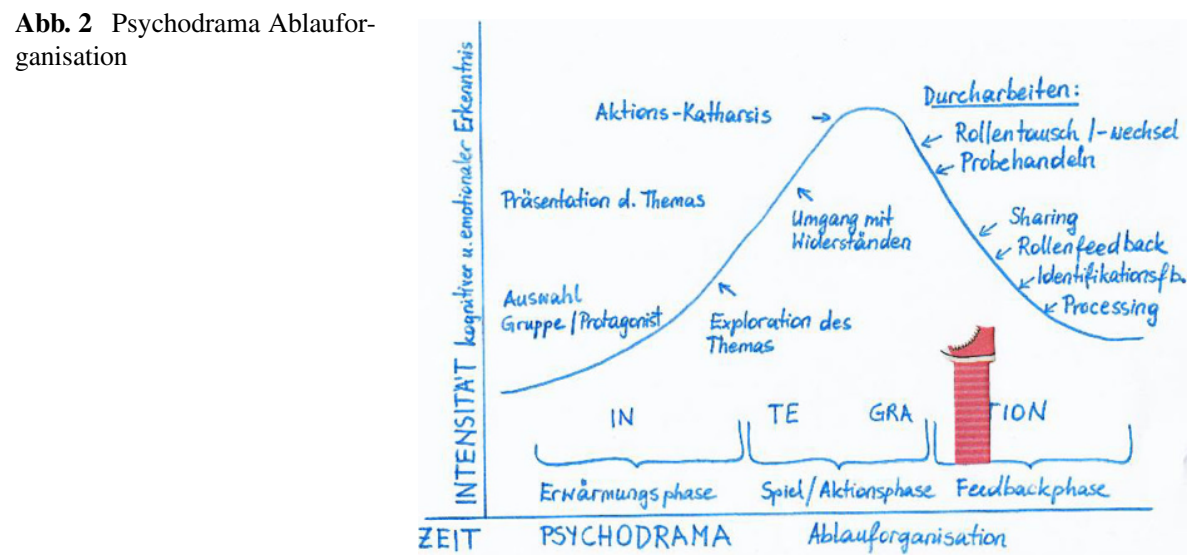


stellen konnte, wenn er oder sie Zeit dafür hatte. Einer der Nachteile bestand darin, dass die Reaktionen oft verzögert oder zeitlich versetzt ankamen und inzwischen schon weitere Beiträge in die Gruppe gestellt worden waren.

Es ist für die Gruppenleitung daher eine große Herausforderung, die Inputs der TeilnehmerInnen immer wieder aufzugreifen und fokussiert zu beantworten. Ein längerer Dialog mit wiederholtem Antworten der DialogpartnerInnen auf ein und dasselbe Thema, wie wir es vom direkten Gesprächskontakt her kennen, wodurch eine Vertiefung der Themen zustande kommt, war so nur sehr schwer möglich und kam seltener vor.

Ein weiterer Nachteil ist die Schwierigkeit der zeitlichen Strukturierung. Für die Einreichung von Seminarinhalten, wie zum Beispiel Warm-Up-Inputs und die Beendigung des Seminars mussten Termine gesetzt werden.

Eine weitere Herausforderung beim Instant-Messaging ist die, dass viel geschrieben wird und teilweise durch die Autokorrektur der Applikation, oder teilweise durch Konzentrationsfehler der Schreibenden immer wieder fehlerhafte Texte geschickt werden. - Fehlerfreundlichkeit und Fantasie beim Leser oder der Leserin sind daher gefordert. Unter Umständen ist genau das auch die Schwierigkeit für Menschen mit Lese-Rechtschreibschwäche, sich mithilfe dieses Mediums auszudrücken. Zum Glück gibt es da auch die Möglichkeit der Sprach- oder Videonachricht, um ohne allzu große Anstrengung, Beiträge in die Gruppe zu stellen. Der eigene Ausdruck ist wie Labatzki (2015) bereits über die Mail- oder Chat-Beratung hervorhebt, zum größten Teil über verschriftlichte Sprache möglich, emotionaler Gehalt kann zwar durch Emoticons (,Smileys“") angereichert werden, der Charakter einer leibhaftigen Kommunikation mit ihren vielfältigen Ausdrucksformen kann jedoch auch dadurch nicht erreicht werden. Für die Wahrnehmung des Gegenübers kann vorrangig der visuelle Sinneskanal durch Lesen der Nachricht genutzt werden. Die ,analogen“ Elemente der Kommunikation wie das Hören, die Intonation, die Gestik und Mimik des Gegenübers sind nur über die Audio- oder Videofunktion eingeschränkt erlebbar, der Geruch, Geschmack, körperliche Berührung und vieles mehr fallen weg.

Diese Reduktion bietet jedoch auch Vorteile. Die Konzentration auf die Bedeutung des Geschriebenen wird verschärft und die Fantasietätigkeit beim Leser oder der Leserin wird erhöht, man liest verstärkt ,zwischen den Zeilen“. Ähnlich wie bei der therapeutischen Gegenübertragung in der Psychoanalyse werden die eigenen Gefühle, Körperempfindungen und Gedanken zum Verstehen des Gegenübers genutzt, nur eben noch fokussierter, weil reduzierter. PatientInnen, die sich nicht gern zeigen, fühlen sich dadurch besser geschützt und haben daher häufig weniger Hemmungen, den Kontakt zum Hilfsangebot zu suchen.

Die Gruppenleitung ist im digitalen Setting sicherlich sehr aktiv und fragt viel nach, sucht nach fehlenden Rückmeldungen und möglicherweise ,,verlorenen Schäfchen“. Insgesamt fühlt sich diese Form der Gruppenleitung mit digitalen Medien aus LeiterInnensicht vermehrt nach „Fischen im Trüben“ an. - Das ist zwar in der Selbsterfahrung und auch in der Psychotherapie nichts Neues, aber die Intensität dessen ist verstärkt und die Präsenz der Leitung in ihrer Rolle ist auf diese Art stärker nötig, als beim konventionellen Setting.

Es muss auf klare zeitliche Vorgaben und Rahmenbedingungen geachtet werden, da sonst die Gefahr besteht, dass die Motivation der GruppenteilnehmerInnen verlo- 
rengeht. Es wurde immer wieder klargestellt, wann ein offizieller Seminarcharakter beginnt und wann er endet.

Die zeitlichen Fristsetzungen dürfen dabei nicht über eine Woche hinausgehen, da sonst die Verbindlichkeit der Kommunikation untereinander verloren geht und sich private Inhalte mit Seminarinhalten mischen. Die Instant Messaging Gruppe wurde in der Zwischenzeit von den einzelnen Gruppenmitgliedern je nach Bedarf für die Begleitung durch ihren Alltag genutzt.

Schwierig bei der asynchronen Kommunikation in der Instant Messaging Gruppe war, die Beantwortung der einzelnen Inputs, die oft schon einige Tage zurücklagen während andere aktuell neue Themen einbrachten. Dadurch veränderte sich die Gruppendynamik bildhaft gesprochen hin zu einem Wildbach, bei dem die Fließgeschwindigkeit des Wassers an verschiedenen Stellen unterschiedlich schnell ist (Abb. 3).

Dieses Fließen des Gruppengeschehens verlangte nach Strukturierung. Daher wurde, sobald es möglich war, das zweite Seminar per Videomeeting wie oben beschrieben begonnen und beendet. Diese Intervention sicherte die Klarheit über den zeitlichen Rahmen der Seminararbeit und in welchen Zeiträumen sich die Gruppe außerhalb des Seminars freiwillig, sozusagen ,privat“ per Instant Messaging traf.

Der Gruppenprozess, der entsprechend dem Phasenmodell nach Tuckman (1965) vor der Covid-19-Krise bereits in der Phase des Performings angekommen war,

Abb. 3 Foto Blühnbach. (Ulrike Altendorfer-Kling 2020)

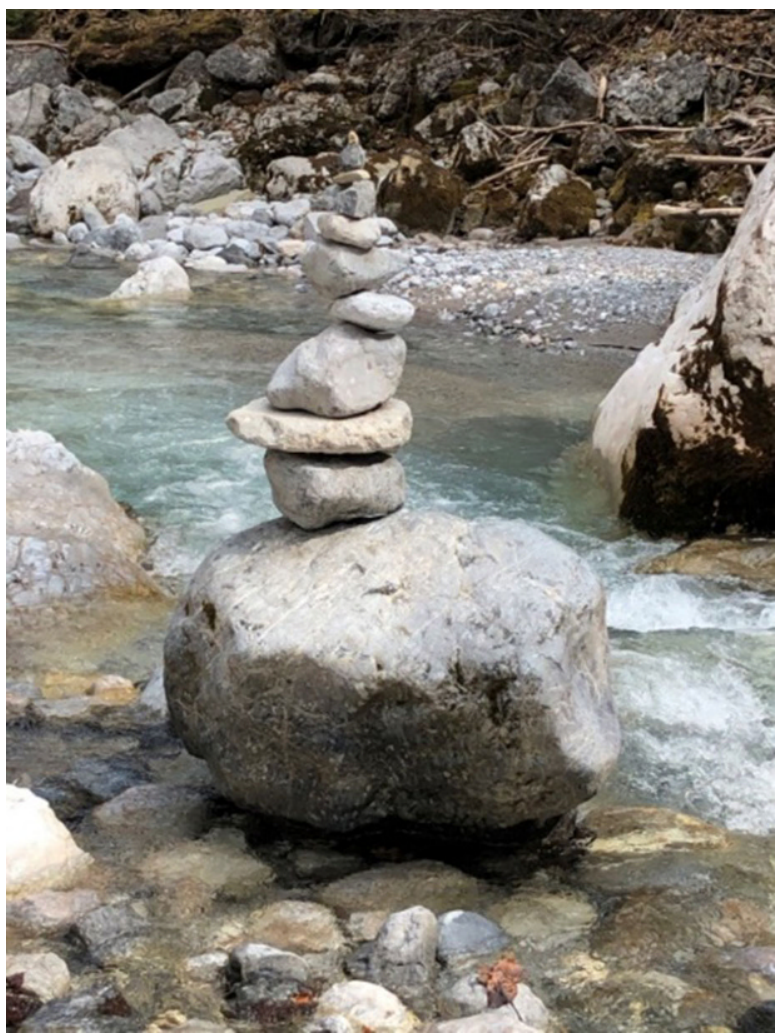


verlief nun in einer neuerlichen Phase des Formings und bald auch des Stormings. Die Gruppe wurde in dieser Phase durch konkrete Theorieinputs, Information und geführte Diskussionsangebote in der Integrationsrunde durch die Leitung stabilisiert.

Techniken wie der Rollenwechsel können per Videotelefonie oder -meetings in der Gruppe in Form der Arbeit mit Symbolen auf der Tischbühne im Raum des oder der TherapeutIn leicht durchgeführt werden, indem der oder die TherapeutIn stellvertretend den Finger auf ein Symbol legt und den oder die ProtagonistIn aus der entsprechenden Rolle heraus sprechen lässt. Das unmittelbare und ganzheitliche Erleben der Arbeit auf der Tischbühne wird für die ProtagonistInnen allerdings dann intensiver, wenn sich die Tischbühne mit den Symbolen in deren Raum befindet und sie die Symbole, unterstützt durch die Fragen des oder der TherapeutIn, in der eigenen Umgebung selbst auswählen und danach auch noch selbst die Symbole berühren und aus den entsprechenden Rollen heraus sprechen können. Diese Form der Arbeit verlangt nach Absprache zwischen TherapeutIn und PatientIn oder Gruppenmitgliedern eventuell einigen Vorbereitungen vor der geplanten Sitzung. Per Videotelefonie erhält der oder die TherapeutIn andere Eindrücke von der Situation und der unmittelbaren Umgebung der PatientInnen oder Gruppenmitglieder in ihrem privaten Umfeld, es können bestimmte Dinge oder das eigene Haustier gezeigt oder versteckt werden, während der oder die PatientIn sich frei und unmittelbar in seinem bzw. ihren Umfeld bewegt. So ist eine andere Form der Begegnung möglich, obwohl meist einige Kilometer in der Realität zwischen PatientIn und TherapeutIn liegen.

Es hat sich bewährt, soziometrische Techniken, wie z.B. das Soziogramm oder das Soziale Atom per Videomeeting auf einem Flipchart zu präsentieren, die Gruppenmitglieder ihre Position zum entsprechenden Kriterium beschreiben zu lassen und sie dann mittels kleiner Notizzettel anzubringen.

Die digitalen Medien können den persönlichen Kontakt und die gegenseitige spontane Körperwahrnehmung über das Riechen nicht ersetzen. Als verfügbare Sinne stehen das Hören und Sehen zur Verfügung, also der Fokus liegt stärker auf der Stimmmodulation oder den visuellen Eindrücken in einem begrenzten Blickfeld, aus dem sich TherapeutIn und Gruppenmitglied unvermittelt heraus- oder hineinbewegen können. Eine klare Körpersprache und vor allem die bewusste Mimik wird geschult.

\section{Diskussion}

Die Entwicklung der digitalen Medien als Tools für die Psychotherapie steckt derzeit noch in den Kinderschuhen. Einen wichtigen Impuls hat die Covid-19-Krise geliefert. Die Einsatzmöglichkeiten in den verschiedenen Bereichen der Psychotherapie und Beratung sind derzeit gekennzeichnet von vielversprechenden Erweiterungen des Angebots und andererseits von Beschränkungen durch die aktuellen technischen Möglichkeiten.

Der Kunstbegriff der „Digitalen Heilung“ repräsentiert laut Labatzki (2020) die dialektische Sichtweise, die sich wie ein roter Faden durch das Thema zieht. Digitale Heilung kann verstanden werden als Heilung von den gesundheitlichen Folgen der 
Digitalisierung oder als Heilung durch digitale Medien bei gesundheitlichen, in diesem Falle psychischen Störungen und Erkrankungen.

Das Internet kann als Informationsmedium bei der eigenen Recherche über psychische Störungen helfen und mittels qualifizierter Selbsttests sogar diagnostische Hinweise liefern.

Der niederschwellige Zugang reduziert Schwierigkeiten wie beispielsweise die Angst vor Stigmatisierung oder Schamgefühle. Das Internet ermöglicht zudem Formen der Nachsorge auch nach stationärer Therapie und erfüllt eine „Brückenfunktion" sowohl in der Vor- als auch in der Nachsorge (Stepped Care). Auch als ergänzendes Angebot von Face-to-Face-Therapie oder Beratung (Blended Care) kann sich internetbasierte Psychotherapie als wirksam erweisen.

Als Nachteile der Beratung und Therapie im Internet führt Labatzki (2015) an:

Im Netz tummeln sich neben vielen seriösen und erprobten Beratungsangeboten auch reichlich unzuverlässige Seiten, deren Nutzung eventuell mehr schadet als hilft. Die Unterscheidung fällt den Ratsuchenden oft nicht leicht.

In schweren Krisensituationen, beispielsweise bei akuter Suizidalität, ist meist sicherlich eine direkte menschliche Begegnung eher angezeigt als ein Gespräch per Bildschirm oder Tastatur. Besonders in solchen Fällen kann es zusätzlich zum Problem werden, dass die Behandlung gerade infolge ihrer Niederschwelligkeit so einfach abgebrochen werden kann. Die flexible Strukturierung der Angebote trägt nicht unbedingt zu einer Sicherung der Compliance bei den Ratsuchenden bei, und auch die Klärung von eventuellen Irritationen und Missverständnissen kann für den Beratenden schwierig werden. Nicht zuletzt ist die Einhaltung datenschutzrechtlicher Bestimmungen nicht immer zuverlässig nachprüfbar, und uneinheitliche Qualitätsstandards machen die Beurteilung der ohnehin unübersichtlichen Angebote zusätzlich schwierig.

Labatzki (2020) fasst wie folgt zusammen: Die übermäßige Beschäftigung mit der digitalen Welt kann auch Folgen nach sich ziehen, die unmittelbar im medizinischen und/oder psychosozialen Bereich anzusiedeln sind. Auf der individuellen physischen Ebene sind zum Beispiel Sehstörungen, Schlafstörungen, Haltungsschäden, Unfälle, Adipositas zu nennen, auf der psychischen Ebene Konzentrationsprobleme, Leistungsstörungen, Depressionen, soziale Phobien, Onlinesucht oder pathologischer Medienkonsum. Soziale Folgen können beispielsweise Vereinsamung, Partnerschaftsprobleme, familiäre Probleme, Probleme durch Cybermobbing und „Shitstorms“ sein. Die Nutzung von Metadaten kann das Kaufverhalten des Einzelnen manipulieren oder sogar zu politischer Radikalisierung führen (Stichwort „Fake News“).

Die digitalen Medien verändern die Lebensweise der Menschen radikal, und es ist noch offen, ob diese Entwicklung eher Segen oder Fluch werden wird, je nachdem wie wir Menschen damit umgehen.

Schon jetzt sind sie nicht mehr aus der Psychotherapiepraxis wegzudenken. Das Handy wird häufig von PatientInnen gebracht, mit der Bitte, es während der Stunde aufzuladen. Ich lege meines bewusst aus dem Praxisraum hinaus, damit stimmenaktivierte virtuelle Assistenten nicht meine Gespräche mit PatientInnen mithören können und ich meinem Schweigepflichtgebot und den Anforderungen des Datenschutzgesetzes nachkommen kann. Wir nutzen die Foto- und Videofunktion häufig, 
um Symbolarbeiten zu dokumentieren, Slow-Motion- oder andere Videos zu drehen oder wir speichern positive Affirmationen als Erinnerungsfunktionen ein. Auch während der Videotelefonie nutze ich immer wieder die Screenshot-Funktion, um bedeutsame Momente festzuhalten und zu spiegeln. Die Patienten freuen sich danach, wenn sie Fotos aus der Sitzung geschickt bekommen und so Selbstbewusstseinsstärkung über die digitale Spiegeltechnik erfahren.

In der beschriebenen Selbsterfahrungsgruppe wurden die digitalen Medien im Sinne von Intermediärobjekten eingesetzt, um bestehende Beziehungen in der Gruppe aufrechtzuerhalten, es war schließlich nicht absehbar, wie lange es dauern würde, bis sich die Gruppe wieder treffen können wird.

Daher wurde eine Begleitung und Resilienzförderung innerhalb der Gruppe durch die aktuelle Krise mittels digitaler Medien angeboten, die von den Gruppenmitgliedern unterschiedlich angenommen wurde. Einige Mitglieder freuten sich über das Angebot der blended Care durch die Instant Messaging Gruppe und nützten diese für sich, um auch privat mit den anderen Gruppenmitgliedern in Kontakt zu bleiben. Andere bevorzugten die Unterstützung durch die Videokonferenzen, da diese einen klarer abgegrenzten zeitlichen Rahmen boten und wieder andere konnten mit dem Angebot zunächst wenig anfangen, weil sie noch um die bisher gelebten Begegnungsmöglichkeiten mit Face-to-Face-Kontakt und Rollenspielen mit allen Sinnen trauerten und sich auf diese plötzliche Veränderung der Begegnungsangebote nur schwer einstellen konnten.

Im Ausbildungskontext sind die digitalen Medien im 21. Jahrhundert in kürzester Zeit wichtige Lehrmittel und Lehrinhalte geworden, sodass die Studierenden, die ja häufig schon damit aufgewachsen sind, nun auch deren therapeutischen Einsatz erlernen. Zusätzlich sind Aufklärung, Information und Bildung in Familie und Schule unverzichtbar.

Medienkompetenz gehört nach Labatzki (2020) zu den beruflichen und persönlichen „Skills“ des postmodernen Menschen. Dementsprechend gehört die altersgemäße Heranführung an diese Medien zum Bildungsauftrag.

\section{Fazit}

Wir alle hoffen, bald wieder die gewohnte Form der Gruppentreffen leben zu dürfen, haben aber mit unserer neuen Form der Zusammenarbeit einen wichtigen Schritt gesetzt, um Psychodrama krisenfest zu machen und Morenos Ansatz der gelebten Kreativität und Spontaneität zu folgen.

Labatzki (2020) berichtet, dass $83 \%$ der 14- bis 29-Jährigen in Deutschland mindestens wöchentlich Videoportale wie YouTube nutzen. Bei Facebook sehen in dieser Altersgruppe $47 \%$ mindestens wöchentlich Videos. Was auf diesen Plattformen passiert, was dort ein Publikum erreicht, ist also alles andere als irrelevant.

Daher macht es aus meiner Sicht keinen Sinn, sich vor den aktuellen Entwicklungen zu verschließen. In der virtuellen Welt treffen Menschen aus unterschiedlichen Gründen aufeinander, unter anderem um miteinander zu spielen.

Das Internet wird laut Labatzki (2020) zunehmend ein nützliches Medium für Beratung und Therapie (,Telemedizin“) in Deutschland. Krankenkassen bieten dort 
Selbsthilfeprogramme an, Kliniken Modellversuche für Behandlungskonzepte. Der Deutsche Ärztetag hat im Juli 2018 das Fernbehandlungsverbot gelockert. Es gibt Angebote für Online- und Chatberatung und für Psychotherapie von Beratungsstellen und von TherapeutInnen. Evaluationsstudien belegen die Wirksamkeit dieser neuen Behandlungsformen.

In Österreich wurde erstmals 2020 für die Zeit der Covid-19-Krise Psychotherapie und verschiedene ärztliche Leistungen über digitale Medien erlaubt und diese Leistungen von den Sozialversicherungsträgern bezahlt.

Blended Care kombiniert das Beste beider Welten - in unserem Fall die Welt der Live-Psychodramatherapie und der virtuellen Welt der digitalen Medien. Silbermayr (2010) stellt Bezüge zwischen Online-Rollenspielen und der Psychodramabühne her.

Ich bin zuversichtlich, dass die Integration der digitalen Medien in der Psychodrama Therapie und im Ausbildungskontext sowohl für unsere KlientInnen und PatientInnen als auch für uns als TherapeutInnen und ÄrztInnen gelingen wird und PsychodramatikerInnen die digitale Revolution mitvollziehen können, indem sie nach und nach weitere Tools erarbeiten und zur Verfügung stellen.

\section{Literatur}

\section{Verwendete Literatur}

Labatzki, U. (2015). (Psychodynamische) Psychotherapie im Zeitalter des Internets. Psychodynamische Psychotherapie, 14(1), 27-36.

Labatzki, U. (2020). Digitale Infekte - Digitale Heilung. In K. Heiland (Hrsg.), Prinzip Infektion, Atmosphärische Übertragung in Gesellschaft, Kunst und Psychoanalyse. Gießen: Psychosozialverlag. Im Erscheinen.

Shakespeare, W. (vermutlich 1599). Theaterstück All the world's a stage, Wie es euch gefällt - As You Like It, 2. Akt, 7. Szene / Original engl. „All the world's a stage, and all the men and women merely players“. Uraufführung 1603

Silbermayr, E. (2010). Die virtuelle Welt als Psychodrama Bühne, Begegnung mit dem Avatar. Zeitschrift für Psychodrama und Soziometrie, 9, 269-280.

Stadler, C., Spitzer-Prochazka, S., Kern, E., \& Kress, B. (2018). Act creative! Effektive Tools für Beratung, Coaching, Psychotherapie und Supervision (S. 233-237). Stuttgart: Klett-Cotta.

Tuckman, B. W. (1965). Developmental Sequences in small groups. Psychological Bulletin, 63, 348-399.

\section{Weiterführende Literatur}

Labatzki, U. (2021). Entwicklungen in der Onlineberatung - von der Präsenzberatung zur „hybriden“ Beratung. erscheint 2021 im Kongressreader zum 17. Bonner Symposium der KBAP, September 2019: Global - digital - vernetzt: Quo vadis, Psychotherapie? 


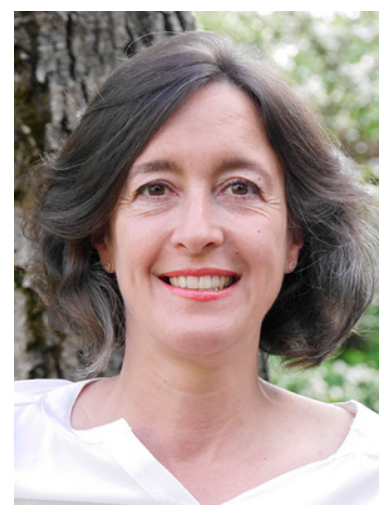

Dr. med. univ. Ulrike Altendorfer-Kling 1972, Fachärztin für Kinderund Jugendpsychiatrie und Psychotherapeutische Medizin, Ärztliche Leiterin der Kinder-Jugend-Seelenhilfe Salzburg, Lehrende an der Paracelsus Medizinischen Privatuniversität Salzburg, Lehrtherapeutin mit partieller Lehrbefugnis für Psychodrama, Soziometrie und Rollenspiel (ÖAGG), Lehrbeauftragte für Psychosoziale, Psychosomatische und Psychotherapeutische Medizin der ÖÄK, Mitglied der ÖGKJP, Psychodrama Psychotherapeutin in freier Praxis mit Weiterbildung in Säuglings-, Kinder- und Jugendlichenpsychotherapie 